\title{
PDE4D haploinsufficiency syndrome
}

INSERM

\section{Source}

INSERM. (1999). Orphanet: an online rare disease and orphan drug data base. PDEAD haploinsufficiency syndrome. ORPHA:439822

PDE4D haploinsufficiency syndrome is a rare syndromic intellectual disability

characterized by developmental delay, intellectual disability, low body mass index, long arms, fingers and toes, prominent nose and small chin. 